\title{
STIMULATED-EMISSION-PUMPING LASER-INDUCED-FLUORESCENCE SPECTROSCOPY OF PHENOL AND ANISOLE
}

\author{
MASAO TAKAYANAGI and ICHIRO HANAZAKI \\ Institute for Molecular Science, Myodaiji, Okazaki 444, Japan
}

(Received 10 April 1993)

\begin{abstract}
The SEP-LIF (stimulated emission pumping-laser induced fluorescence) technique was applied to the investigation of dynamical behavior of vibrationally excited phenol and anisole produced in the supersonic expansion. In the SEP-LIF scheme, a molecule excited to a specific vibrational state by SEP is detected by measuring the LIF excitation spectrum with an appropriate delay to probe the vibrational relaxation. Four vibrational states, $6 \mathrm{a}_{1}, 16 \mathrm{a}_{2}, 12_{1}$ and $1_{1}$, of phenol, and six vibrational states, $18 \mathrm{~b}_{1}, 18 \mathrm{~b}_{2}, 6 \mathrm{a}_{1}, 12_{1}$, $16 \mathrm{a}_{2}$ and $1_{1}$, of anisole were investigated. For both of phenol and anisole, it is found that the relaxation of the vibrational states below $1,000 \mathrm{~cm}^{-1}$ in the ground electronic state is so slow under the collisionless condition that only the transitions from the vibrational states initially prepared by SEP are observed as the SEP-induced bands in the SEP-LIF spectra. The low frequency torsional motion of methyl group in anisole does not accelerate IVR (intramolecular vibrational redistribution) much in this energy region.
\end{abstract}

KEY WORDS: Phenol; anisole; SEP; LIF; IVR.

\section{INTRODUCTION}

It has been pointed out that the collisionless IVR (intramolecular vibrational redistribution) rate is accelerated by low frequency vibrations. The fast IVR in the excited electronic states of alkyl-substituted aromatic molecules has been studied extensively with the measurements of single-vibronic dispersed fluorescence spectra, ${ }^{1}$ the fluorescence decay measurements, ${ }^{2}$ the time-resolved photoelectron spectroscopy ${ }^{3}$ and the chemical timing technique., ${ }^{4,5}$ On the other hand, less studies have been reported for the IVR in the ground electronic state.

Information on the IVR rate in the ground electronic state has been obtained with a pump-probe technique, ${ }^{6}$ and from the analysis of the depths of dips in the stimulated-emission-pumping ion-dip spectra of alkylphenols, alkylanilines and trans-stilbene. ${ }^{7,8}$ These are considered to be indirect observations in the sense that the fate of the initially prepared vibrational states is not observed directly. On the other hand, Ebata and Ito ${ }^{8}$ have attempted a direct observation of IVR in the alkylsubstituted aromatics. They measured the decay of vibrationally excited states of 
alkylphenols and alkylanilines with the stimulated Raman-ultraviolet optical double resonance technique. They have found that the decay rates obtained with this technique and those from the analysis of the fluorescence dip spectra are inconsistent with each other. They measured only the decay of the $1_{1}$ states by monitoring the $1_{1}^{0}$ transitions in their double resonance technique. It seems to be required to investigate the excited energy dependence of the decay rate, and to measure the spectra of the excited species in wider frequency range to confirm whether the observed species is certainly in the initially prepared state.

We have applied the SEP-LIF (stimulated emission pumping-laser induced fluorescence) technique to van der Waals complexes containing aromatic molecules. . $^{9-12}$ The decay rates of vibrationally excited benzonitrile•Ar and benzonitrile $\bullet \mathrm{H}_{2} \mathrm{O}$ have been determined, which reflect the rates of IVR or dissociation process. ${ }^{9}$ We have also measured SEP-LIF spectra of the benzonitrile dimer ${ }^{9}$ and the anisole•benzene complex. ${ }^{12}$ The SEP-LIF signal due to the complexes in the initially prepared vibrational states could not be observed even when the energy of the excited vibrational state is below the dissociation threshold of the van der Waals bond. Non-observation of the SEP-LIF signal suggests the existence of fast IVR. It is of interest to see whether this kind of fast IVR is characteristic of complexes or common in both complexes and monomeric molecules.

In view of these, we report in this paper SEP-LIF spectra of phenol and anisole in the supersonic expansion to investigate the dynamics of their excited vibrational states in the ground electronic state. Anisole has a low frequency torsional motion due to the methyl group which is absent in phenol. It is interesting to see how the IVR rate depends on the state density in phenol and anisole.

The SEP-LIF technique employed here is a kind of triple-resonance spectroscopy in which the species excited to a specific vibrational state by SEP is probed by LIF. The species in the initially prepared state can be monitored by the LIF technique as well as those produced by the relaxation or reaction process. The decay rate of the initially prepared state is also measured by varying the delay between the SEP and LIF pulses.

\section{EXPERIMENTAL}

Experimental details have been described elsewhere. ${ }^{9}$ Briefly, frequency doubled outputs of two dye lasers (Quantel: TDL-50, line width: $0.2-0.5 \mathrm{~cm}^{-1}$ ) simultaneously pumped by the second harmonics of a single Nd:YAG laser (Quantel: YG571C) were used for the SEP excitation; one pumps the molecule to be investigated at the $0-0$ transition and the other dumps it from the $\mathrm{v}^{\prime}=0$ state down to a specific vibrationally excited state in the ground electronic state. The dump frequency was determined by measuring fluorescence dip spectra. The power of pump and dump pulses was $\sim 0.2$ and $\sim 1 \mathrm{~mJ} /$ pulse, respectively. The third laser source generated by frequency doubling of the output of an excimer-laser-pumped dye laser (Lumonics: HE-420-SM-B and Lambda Physik: FL3002, $\sim 0.1 \mathrm{~mJ} /$ pulse, line width: $0.1 \mathrm{~cm}^{-1}$ ) 
was used for the LIF measurement. The delay between the SEP excitation and LIF measurement was varied between $300 \mathrm{~ns}$ and $1.3 \mu \mathrm{s}$. LIF measurements with the delay shorter than $300 \mathrm{~ns}$ are difficult because of the interference with a tail of the fluorescence caused by the pump pulse, while the longer limit of the delay measurement is determined by the escaping of the excited species from the region of observation.

Phenol (Katayama Chemical, reagent grade) and anisole (Tokyo Kasei, reagent grade) were used without further purification. They were diluted by helium and expanded into a vacuum chamber from a pulsed nozzle (orifice diameter $=0.8 \mathrm{~mm}$, pulse duration $=1 \mathrm{~ms}$ ) with the total pressure of $2.5 \mathrm{~atm}$. The partial pressures of phenol and anisole were 0.02 and 0.5 Torr, respectively. Most of measurements were performed at $20 \mathrm{~mm}$ downstream from the nozzle, where the effect of collisions is considered to be small. Measurements at $8 \mathrm{~mm}$ downstream were also performed to examine the effect of collisional relaxation of excited molecules. The background pressure in the chamber was held below $10^{-4}$ Torr throughout the measurements.

\section{RESULTS AND DISCUSSION}

\subsection{Phenol}

Figure 1 shows the fluorescence dip spectrum of phenol measured with the pump frequency set at the $0-0$ transition $\left(36,352 \mathrm{~cm}^{-1}\right)$. The abscissa shows the dump fre-

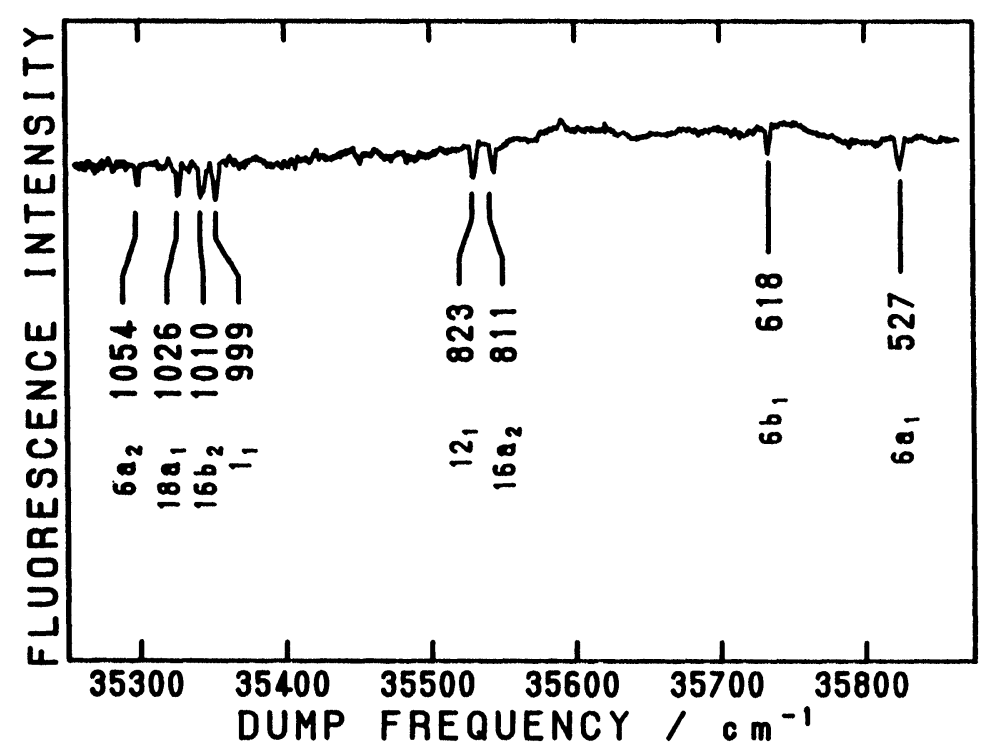

Figure 1 Fluorescence dip spectrum of phenol measured with pumping at the $0-0$ transition $\left(36,352 \mathrm{~cm}^{-1}\right)$. The designation and frequency are shown at each peak. 
quency. The dips observed in the spectrum are not prominent (maximum depletion: $\sim 5 \%$ ). This is presumably because the short lifetime of the excited electronic state of phenol $(\sim 2 \mathrm{~ns})^{13,14}$ makes the effective dumping difficult. The designation and frequency of the vibrational state are indicated in the figure at each peak. We have followed the designations given in the literature. ${ }^{15,16}$

By setting the dump frequency at the peak of one of the dips, the phenol molecule can be excited through the SEP process to a specific vibrational state whose energy corresponds to the difference of the pump and dump frequencies. SEP-LIF spectra of phenol were measured with the excitation of phenol to four vibrational states, $6 \mathrm{a}_{1}$, $16 \mathrm{a}_{2}, 12_{1}$ and $1_{1}$, with the energies of $527,811,823$ and $999 \mathrm{~cm}^{-1}$, respectively.

In Figure 2(a) is shown the LIF excitation spectrum of phenol seeded in He. The

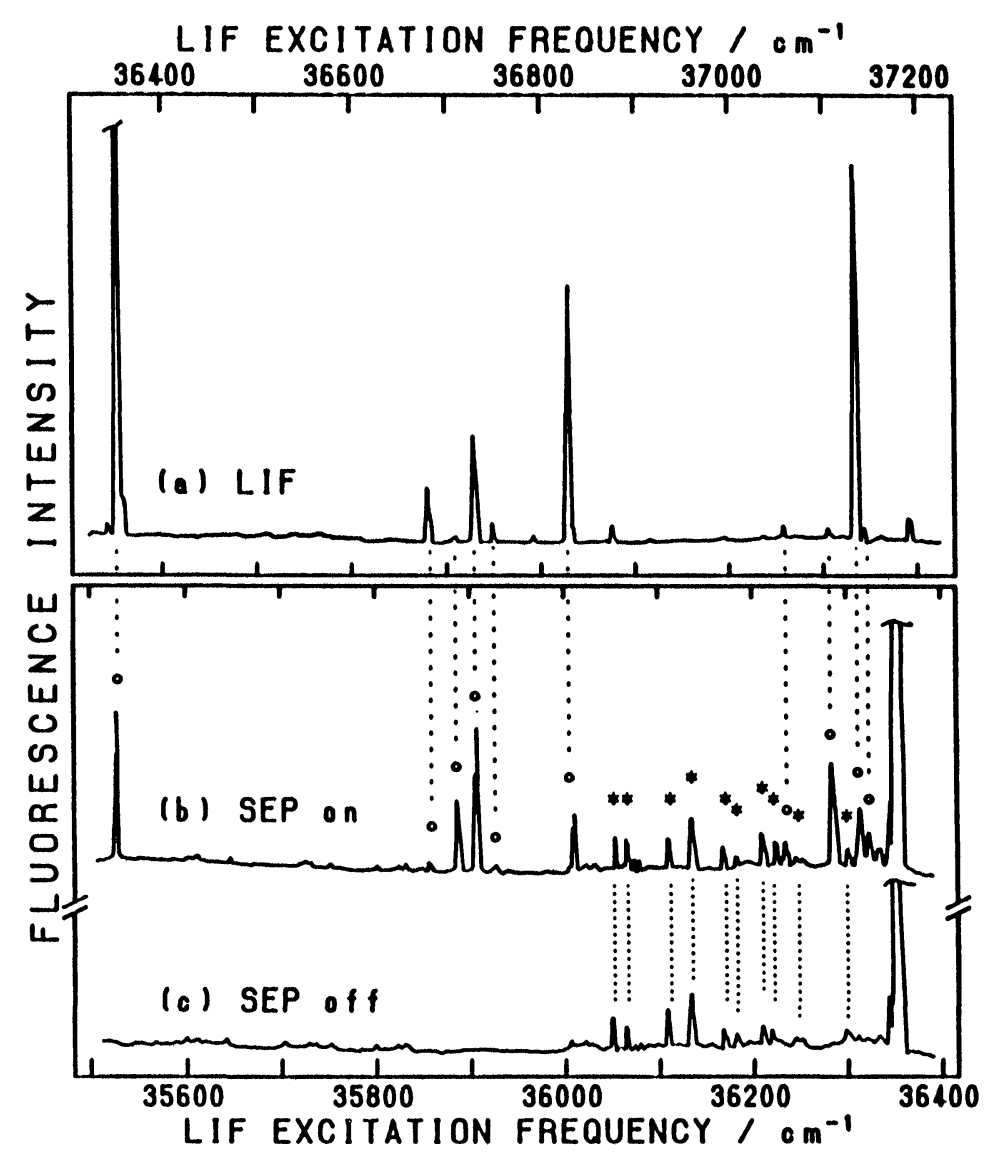

Figure 2 (a) LIF excitation spectrum of phenol (upper scale). (b) SEP-LIF spectrum with the SEP excitation to $12_{1}\left(823 \mathrm{~cm}^{-1}\right)$, and (c) LIF excitation spectrum in the same frequency region as (b). The spectra in (b) and (c) are given with a shift of $823 \mathrm{~cm}^{-1}$ to the right with respect to (a). Thus (c) is the same LIF excitation spectrum as (a) which continues to the left end of (a). The bands marked by open circle in (b) appear upon the SEP excitation. Those with asterisk are the background bands observed without SEP. 
most intense band at $36,352 \mathrm{~cm}^{-1}$ is the $0-0$ band. The bands due to the vibronic transitions of phenol are observed in the higher frequency side.

Figure 2(b) is the SEP-LIF spectrum of phenol measured with SEP to $12_{1}$ $\left(823 \mathrm{~cm}^{-1}\right)$, and with the $300 \mathrm{~ns}$ delay between the SEP and LIF pulses. Figure 2(c) shows the LIF excitation spectrum measured without SEP, which shows the lower frequency region than that in Figure 2(a). The lower scale for Figures 2(b) and 2(c) is shifted from the upper scale for Figure 2(a) to higher frequency by $823 \mathrm{~cm}^{-1}$ in order to make their comparison feasible. The bands observed in the LIF excitation spectrum in the frequency region lower than the $0-0$ band at $36,352 \mathrm{~cm}^{-1}$ [Figure 2 (c)] are due to the hot phenol, the phenol dimer, and the complex of phenol with contaminated water. The corresponding background bands in Figure 2(b) are marked with asterisk.

Some new bands appear upon the SEP excitation as marked with open circle in Figure 2(b). They disappear by turning either the pump or dump pulse off, or by detuning the dump frequency from the SEP resonance. Therefore, the bands should be related to the vibrationally excited phenol. As shown in the schematic energy level diagram in Figure 3, the transition from the vibrational state with the energy of $v_{\text {vib }}$ prepared by SEP to the vibronic level with the energy of $v_{\mathrm{LIF}}$ is observed at the frequency of $v_{\mathrm{SL}}=v_{\mathrm{LIF}}-v_{\mathrm{vib}}$; i.e. if there exists neither relaxation nor dissociation in the vibrational state prepared by SEP, the SEP-induced band and the LIF band with a common upper state should coincide with each other when the former is shifted by the frequency of corresponding vibrational band. For example, the band

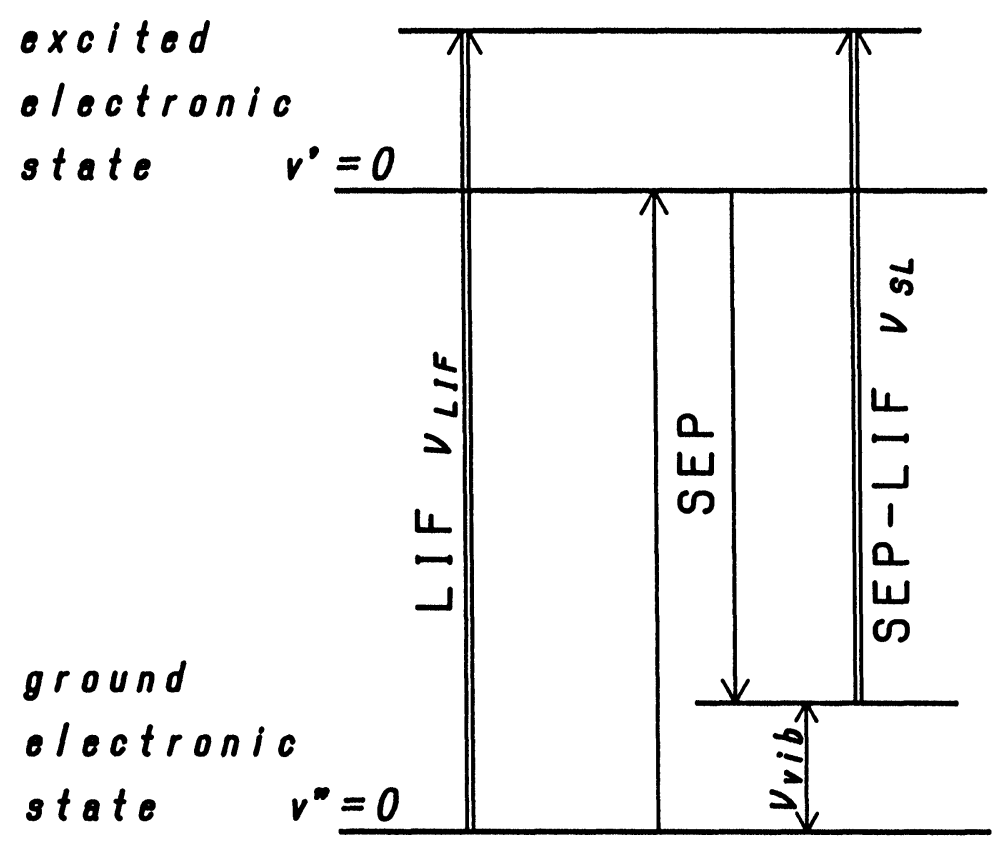

Figure 3 Schematic level diagram showing the relation between the transitions observed in the LIF excitation and SEP-LIF spectra. 
at $35,529 \mathrm{~cm}^{-1}$ in Figure 2(b), whose position agrees with the $0-0$ band at $36,352 \mathrm{~cm}^{-1}$ in Figure 2(a), is due to the $12_{1}^{0}$ transition.

For each of the SEP-induced band in Figure 2(b), a corresponding band is observed in the LIF excitation spectrum in Figure 2(a) as linked by broken lines. This means that all the SEP-induced bands observed with the $12_{1}$ excitation are due to the transitions from the initially prepared vibrational state. The bands due to the vibrationally relaxed species are not observed. This result suggests that phenol in the $12_{1}$ state does not relax appreciably within the delay of $300 \mathrm{~ns}$ between the SEP and LIF pulses. The considerable difference in relative intensity between the corresponding bands in the SEP-LIF [Figure 2(b)] and LIF excitation [Figure 2(a)] spectra can be attributed to the difference in the Franck-Condon factor arising from the different initial states.

Similar SEP-LIF spectra were measured with the $6 a_{1}, 16 a_{2}$ and $1_{1}$ excitation of phenol. The results are summarized in Figure 4 together with the above-mentioned result for the $12_{1}$ excitation. The delay between the SEP and LIF pulses is $300 \mathrm{~ns}$. In the figure, each spectrum is shifted by the frequency of the initially prepared vibrational state so that the band due to the transition to $0^{\circ}$ locates at the origin of abscissa. Then, the abscissa corresponds to the vibrational frequency in the excited electronic state. The intensities are normalized at the transition to $0^{0}$ for each spectrum.

Figure 4 shows that each of SEP-induced bands has its counterpart in the LIF excitation spectra, except for the band at $584 \mathrm{~cm}^{-1}$ marked by a closed circle in the SEP-LIF spectrum measured with the $16 \mathrm{a}_{2}$ excitation. No corresponding band is observed for this band in the LIF excitation spectrum. However, this band can be assigned to the $16 \mathrm{a}_{2}^{0} 10 \mathrm{~b}_{0}^{1}$, the combination transition from the initially prepared vibrational state. The forbidden transition to $10 \mathrm{~b}^{1}$ from $0_{0}$ presumably becomes allowed by the vibrational excitation to $16 \mathrm{a}_{2}$.

These results show clearly that all the SEP-induced bands observed in the SEP-LIF spectra measured with the $6 \mathrm{a}_{1}, 16 \mathrm{a}_{2}, 12_{1}$ and $1_{1}$ excitations are due to the transitions from the vibrational states initially prepared by SEP. No additional band due to the vibrationally relaxed species appears in the SEP-LIF spectra even with the delay between the SEP and LIF pulses as long as $1.3 \mu \mathrm{s}$. Upon increasing the delay, only the decrease of overall intensity is observed which is due to the escape of the excited species from the region of observation. Therefore, we can conclude that the relaxation rates of vibrational states of phenol below $1,000 \mathrm{~cm}^{-1}$ are very slow. This may be understood on the basis of low state density of phenol in this energy region: $\sim 0.1$ and $\sim 0.2$ states $/ \mathrm{cm}^{-1}$, respectively, for the vibrational energies of 700 and $1,000 \mathrm{~cm}^{-1}$. These values are so low that appreciable coupling between the vibrational states cannot be expected.

SEP-LIF measurements shown in Figure 4 are also useful to check the assignment of bands in the LIF excitation spectrum. The band at $327 \mathrm{~cm}^{-1}$ in the LIF excitation spectrum has been assigned as a hot band in the literature. ${ }^{16}$ However, SEP-induced bands are observed at the same frequency in the SEP-LIF spectra measured with the $6 a_{1}, 16 a_{2}$ and $12_{1}$ excitation shown in Figure 4. Therefore, this LIF band is not 


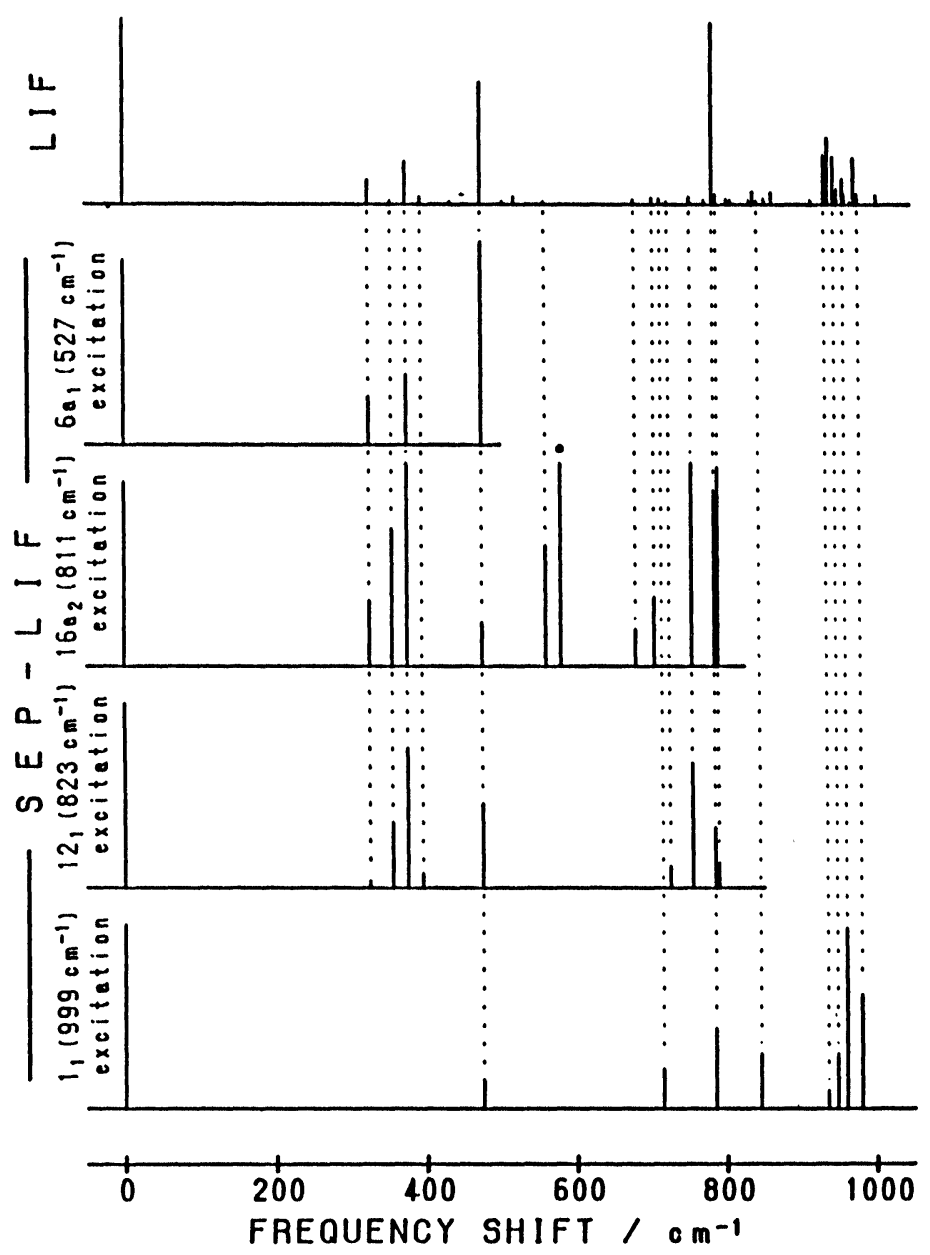

Figure 4 LIF excitation spectrum and SEP-LIF spectra measured with the $6 \mathrm{a}_{1}, 16 \mathrm{a}_{1}, 12_{1}$ and $1_{1}$ excitation of phenol. Spectra are shifted so that the abscissa corresponds to the vibrational energy in the final vibronic states above $0^{0}$ assuming the observed transitions to occur from the initially prepared vibrational state. Intensity scale are relative.

a hot band, but the transition to an unassigned vibronic state locating at $327 \mathrm{~cm}^{-1}$ above the origin of the $S_{1}$ state.

\subsection{Anisole}

In Figure 5 is shown the fluorescence dip spectrum of anisole measured with the pump frequency set at the $0-0$ transition $\left(36,386 \mathrm{~cm}^{-1}\right)$. The designation ${ }^{17-19}$ and frequency of the vibrational state are shown at each peak. The dips are more distinct than those for phenol. The longer lifetime $(23 \mathrm{~ns})^{19}$ of the excited electronic state of anisole makes the dumping from the electronically excited state more effective than 


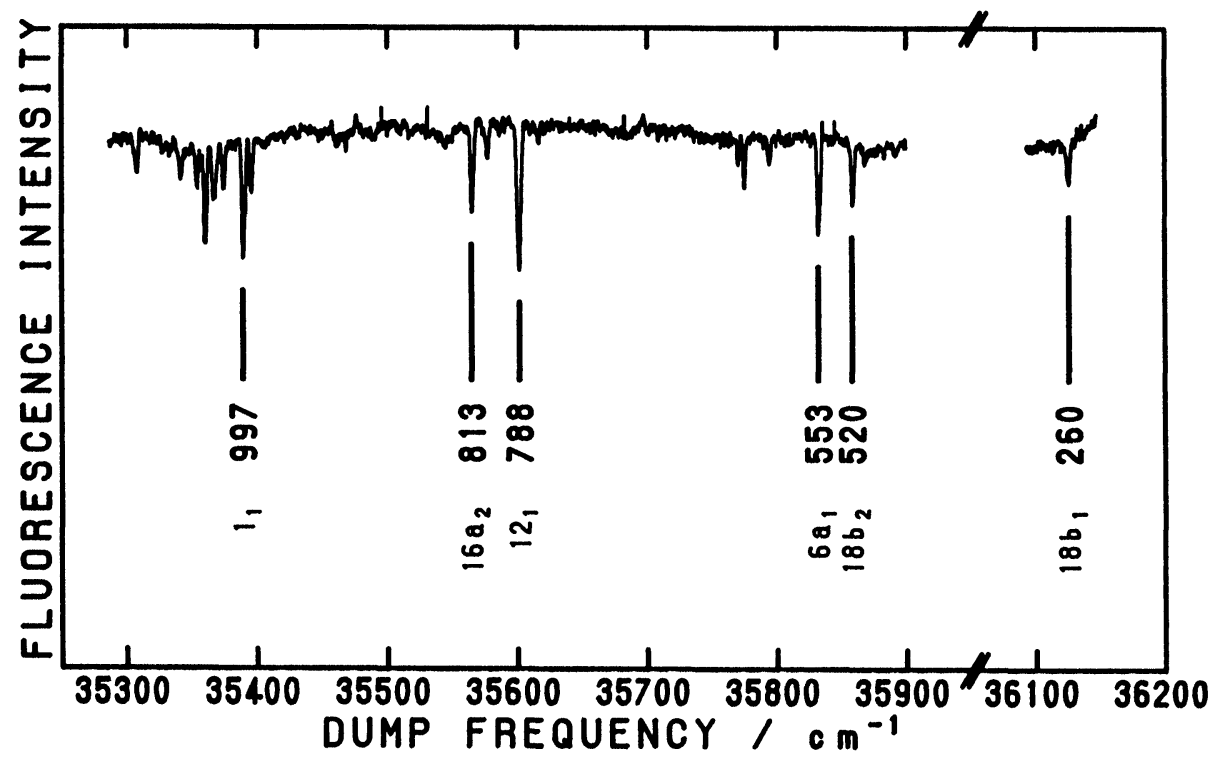

Figure 5 Fluorescence dip spectrum of anisole measured with pumping at the 0-0 transition $\left(36,386 \mathrm{~cm}^{-1}\right)$. The designation and frequency are shown at each peak.

that in phenol. Existence of fast decay (relaxation and/or dissociation) processes in the final vibrational state would also bring about a deeper dip, $7,8,20$ though the dips observed for anisole are not quite remarkable (maximum depletion; $25 \%$ ).

SEP-LIF spectra of anisole were measured with the SEP excitation to six vibrational states, $18 \mathrm{~b}_{1}, 18 \mathrm{~b}_{2}, 6 \mathrm{a}_{1}, 12_{1}, 16 \mathrm{a}_{2}$ and $1_{1}$ with the energies of $260,520,553,788,813$ and $997 \mathrm{~cm}^{-1}$, respectively. Results for the excitation to $18 \mathrm{~b}_{1}, 18 \mathrm{~b}_{2}$ and $6 \mathrm{a}_{1}$ are shown in Figure 6 together with the LIF excitation spectrum. The abscissa shows the frequency taking $\nu_{0}^{0}-\nu_{\text {vib }}$ as the common origin; i.e., the SEP-LIF spectra are shifted by the frequencies of the vibrational states, $v_{\text {vib }}$, prepared by SEP. Bands with asterisk are background bands which are observed without SEP excitation. There exist corresponding LIF bands for most of the SEP-induced bands as linked by broken lines. They can be assigned to the transitions from the initially prepared vibrational states.

No corresponding band in the LIF excitation spectrum is found for the bands marked with closed circle, the $462 \mathrm{~cm}^{-1}$ band for the $18 \mathrm{~b}_{2}$ excitation and the $430 \mathrm{~cm}^{-1}$ band for the $6 \mathrm{a}_{1}$ excitation. They might be due to some relaxed species. However, their relative intensities to the other SEP-induced bands do not depend on the experimental conditions such as the delay between the SEP and LIF pulses, the stagnation pressure and the distance between the nozzle and the point of measurement. Therefore, the bands are most probably due to the species in the initially prepared vibrational states rather than the relaxed species. This is supported by the observation of the $462 \mathrm{~cm}^{-1}$ band in the SEP-LIF spectrum measured with the $786 \mathrm{~cm}^{-1}$ excitation (see below). The 430 and $462 \mathrm{~cm}^{-1}$ vibrational states in the electronically excited state may not be optically accessible from the $0_{0}$ state. 


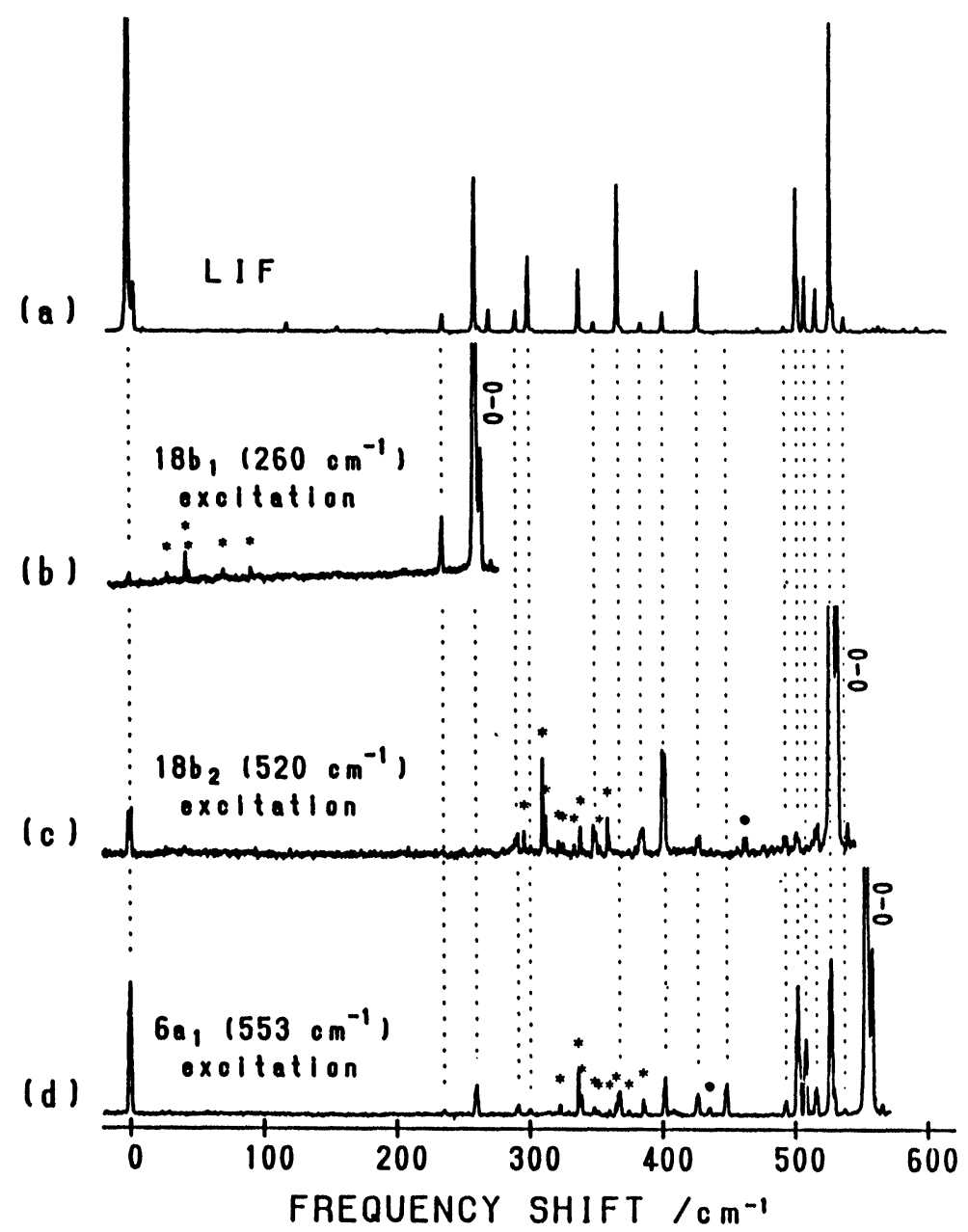

Figure 6 LIF excitation spectrum and SEP-LIF spectra with the $18 b_{1}, 18 b_{2}$ and $6 a_{1}$ excitation of anisole. Spectra are shifted so that the abscissa corresponds to the excess vibrational energy above $0^{0}$ for each excitation. Bands marked with asterisk are the background bands which are observed without SEP excitation. Bands with closed circle appear upon SEP excitation and have no corresponding counterpart in the LIF excitation spectrum. The intense bands marked with $0-0$ are the $0-0$ bands.

The bands due to $18 \mathrm{~b}_{1}^{0}$ and $18 \mathrm{~b}_{2}^{0}$ are observed only weakly at $0 \mathrm{~cm}^{-1}$ in Figures $6(\mathrm{~b})$ and $6(\mathrm{c})$, respectively. Moreover, the $18 \mathrm{~b}_{2}^{1}$ transition, which should appear around $260 \mathrm{~cm}^{-1}$, is not observed. This may be due to low Franck-Condon factors for the $18 b_{1}^{0}, 18 b_{2}^{0}$ and $18 b_{2}^{1}$ transitions. The bands due to $18 b_{1}^{1}$ and $18 b_{2}^{2}$ are probably hidden by the intense $0-0$ band, since the frequency of the $18 \mathrm{~b}$ mode in the electronically excited state is almost the same as that in the ground electronic state. ${ }^{17}$

The $788 \mathrm{~cm}^{-1}$ dip in the fluorescence dip spectrum of anisole (Figure 5) was found to consist of two vibrational states in the ground electronic state. Figure 7 shows 


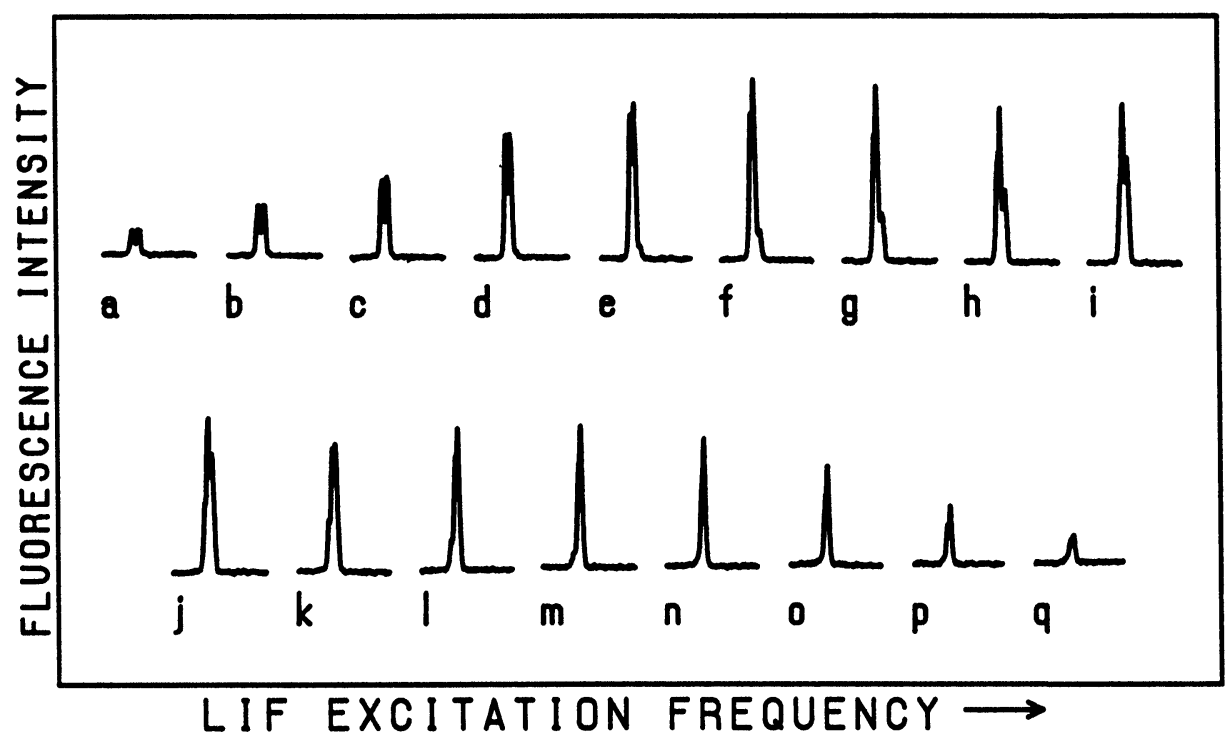

Figure 7 The $12_{1}^{0}$ transition region of the SEP-LIF spectra of anisole with the $\sim 788 \mathrm{~cm}^{-1}$ SEP excitation measured with the different dump frequencies whose interval are $0.003 \mathrm{~nm}\left(0.35 \mathrm{~cm}^{-1}\right)$. The dump frequency decreases from a to $\mathbf{q}$.

the SEP-LIF signal in the $12_{1}^{0}$ transition region measured with the $\sim 788 \mathrm{~cm}^{-1}$ excitation by varying the dump frequency in an interval of $0.003 \mathrm{~nm}\left(0.35 \mathrm{~cm}^{-1}\right)$ from a through q. The dump frequency decreases from a to q. The band in Figure 7 has several peaks, the relative intensities of which vary upon varying the dump frequency. Two intensity maxima at two different dump frequencies ( $\mathbf{f}$ and $\mathbf{m}$ ) indicate the existence of two vibrational states in this region. The intensity maximum at $\mathbf{j}$ is presumably due to the overlap of the $\mathrm{R}$ branch of the lower energy component and the $\mathrm{P}$ branch of the higher energy component, and is not due to the third vibrational state. The two vibrational states with the frequency difference about $2.5 \mathrm{~cm}^{-1}$ will be designated hereafter as the 786 and $788 \mathrm{~cm}^{-1}$ vibrational states, one of which must be the $12_{1}$ state, while the other vibrational state is unassigned. They could not be resolved in the fluorescence dip spectrum because of the fluctuation in the fluorescence baseline.

SEP-LIF spectra are shown in Figure 8 for the 786 and $788 \mathrm{~cm}^{-1}$ vibrational excitations by setting the dump frequency at those corresponding to $\mathbf{o}$ and $\mathbf{d}$, respectively, in Figure 7. Bands marked with asterisk are background bands which are observed without SEP excitation. Many new bands appear upon the SEP excitation. The patterns of the SEP-LIF spectra measured with the 786 and $788 \mathrm{~cm}^{-1}$ excitation are different appreciably; e.g., the 175 and $680 \mathrm{~cm}^{-1}$ bands are observed only in Figure 8(b), while the $262 \mathrm{~cm}^{-1}$ band is observed only in Figure 8(c).

In the region below $550 \mathrm{~cm}^{-1}$, most of the SEP-induced bands observed in Figures 8(b) and 8(c) have corresponding bands in the LIF excitation spectrum shown in Figure 8(a) as linked by broken lines. On the other hand, many bands appear above 


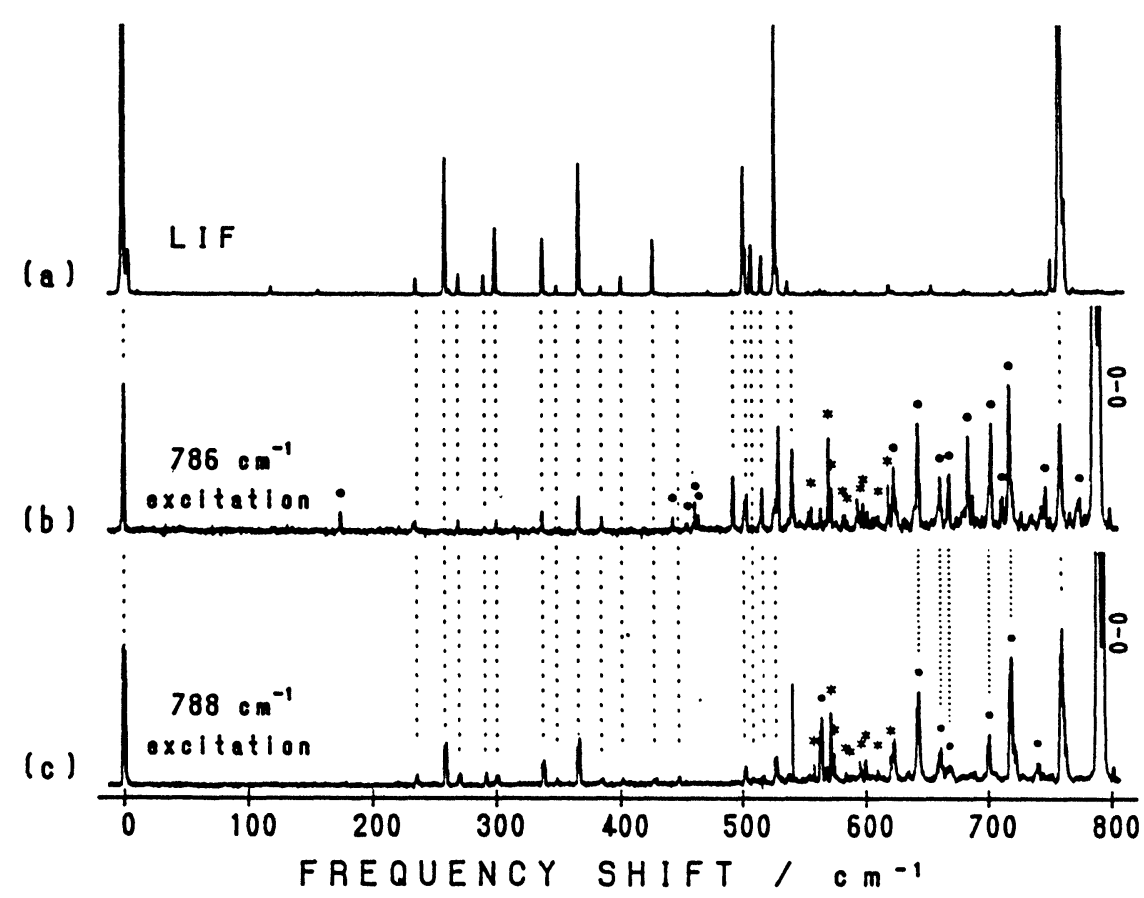

Figure 8 LIF excitation spectrum and SEP-LIF spectra measured with the 786 and $788 \mathrm{~cm}^{-1}$ vibrational excitations of anisole. Spectra are shifted so that the abscissa shows the excess vibrational energy above $0^{0}$ for each excitation assuming the transitions to occur from the initially prepared vibrational state. Bands marked with asterisk are the background bands which are observed without SEP excitation. Those with closed circle appear upon the SEP excitation and have no counterpart in the LIF excitation spectrum. The intense bands marked with $0-0$ are the $0-0$ bands.

$550 \mathrm{~cm}^{-1}$ in the SEP-LIF spectra measured with 786 and $788 \mathrm{~cm}^{-1}$ excitation, which have no counterpart in the LIF excitation spectrum. They are marked with closed circle. Some of them are observed commonly in Figures 8(b) and 8(c) as linked by dotted lines. This suggests that these bands are due to the transitions from the initially prepared vibrational states (the 786 and $788 \mathrm{~cm}^{-1}$ states) to common upper vibronic states. The SEP-induced band at $462 \mathrm{~cm}^{-1}$ in Figure 8(b) has counterpart neither in the LIF excitation spectrum nor in Figure 8(c). However, the corresponding band is observed in the SEP-LIF spectrum measured with the $18 \mathrm{~b}_{2}$ excitation [Figure 6(c)]. Therefore, it should also be due to the transitions from the initially prepared vibrational states (the $786 \mathrm{~cm}^{-1}$ state and $18 \mathrm{~b}_{2}$, respectively) to a common upper vibronic state. The corresponding transition is not observed in the LIF excitation spectrum probably due to a low transition probability from the $0_{0}$ state.

It is also probable that the common SEP-induced bands are due to the IVR from the initially excited vibrational states to a certain common state. In order to examine the possible effect of relaxation, we have performed the measurements with varying delay and nozzle-laser distance. Figure 9 shows the region just below the $0-0$ band of the SEP-LIF spectra measured with the $786 \mathrm{~cm}^{-1}$ excitation. The intense $0-0$ band 


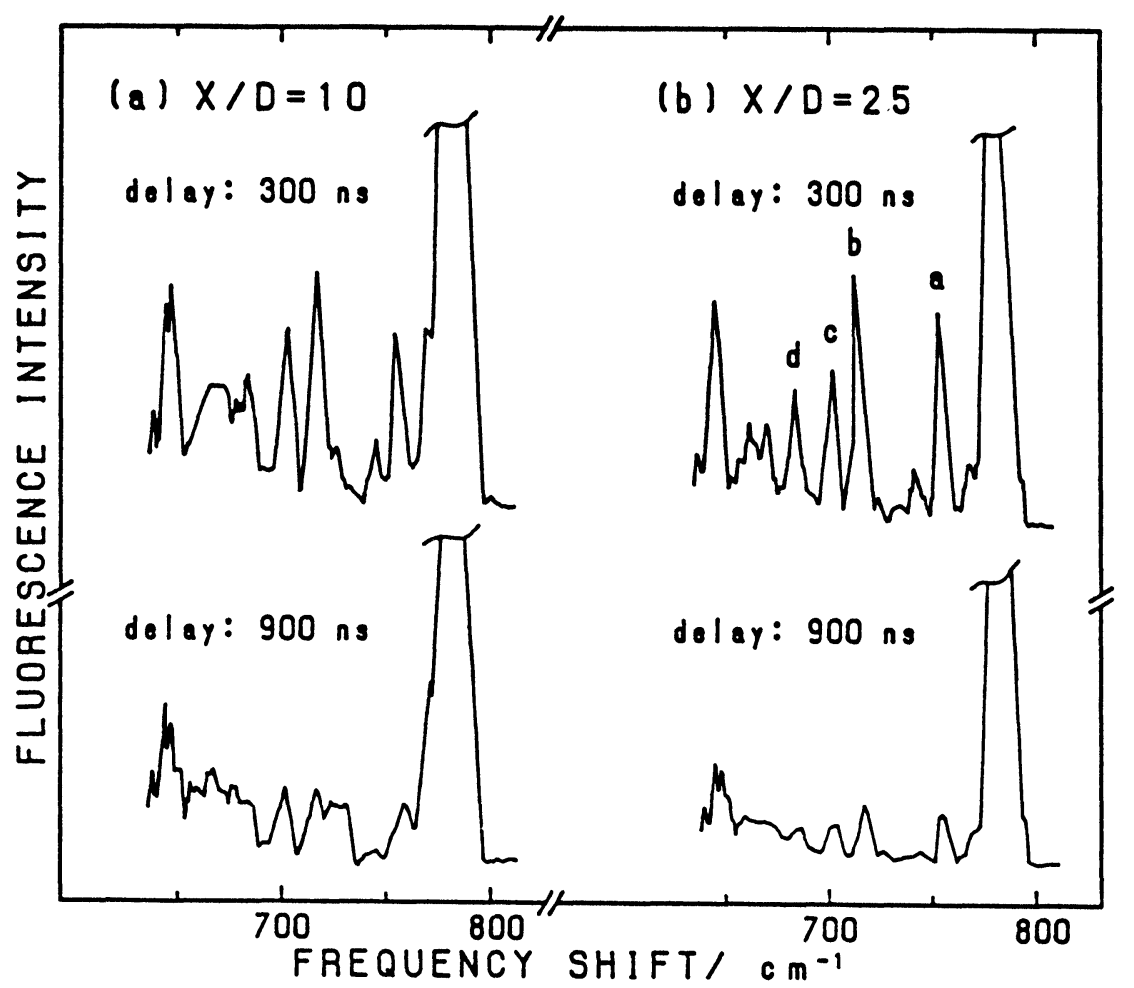

Figure 9 SEP-LIF spectra measured with the $786 \mathrm{~cm}^{-1}$ vibrational excitation of anisole with the pumpprobe delay of 300 and $900 \mathrm{~ns}$. The distance between the nozzle and the point of measurement is (a) $8 \mathrm{~mm}$ and (b) $20 \mathrm{~mm}$. Only the region just below the $0-0$ transition is shown.

is observed at $786 \mathrm{~cm}^{-1}$. At the nozzle-laser distance of $8 \mathrm{~mm}[\mathrm{X} / \mathrm{D}=10$; Figure 9(a)], not only the overall intensity but also the spectral pattern varies upon increasing the delay from $300 \mathrm{~ns}$ to $900 \mathrm{~ns}$. This is presumably due to the collisional relaxation in the high pressure region near the nozzle aperture. On the other hand, at the distance of $20 \mathrm{~mm}[\mathrm{X} / \mathrm{D}=25$; Figure 9(b)], the spectral pattern does not change upon increasing delay from $300 \mathrm{~ns}$ to $900 \mathrm{~ns}$ except for an overall decay which is due to the escape of molecules from the region of observation.

This may be seen more clearly in Figure 10, where the decay of the SEP-induced bands marked with a-d in Figure 9(b) is shown together with that of the SEP-induced band due to the transition to $0^{\circ}$. The decay is measured by varying the delay between the SEP and LIF pulses. The intensities of the SEP-induced bands are normalized at the delay time of $500 \mathrm{~ns}$. In the figure, the same decreasing rates of the band intensities are observed for all the SEP-induced bands, suggesting that the bands are due to the common species. Therefore, the SEP-induced bands with no counterpart in the LIF excitation spectrum in Figure 8 are most probably due to the transitions from the initially prepared vibrational states. Thus the possibility of collision-induced relaxation has been excluded. However, the present result still cannot rule out the 


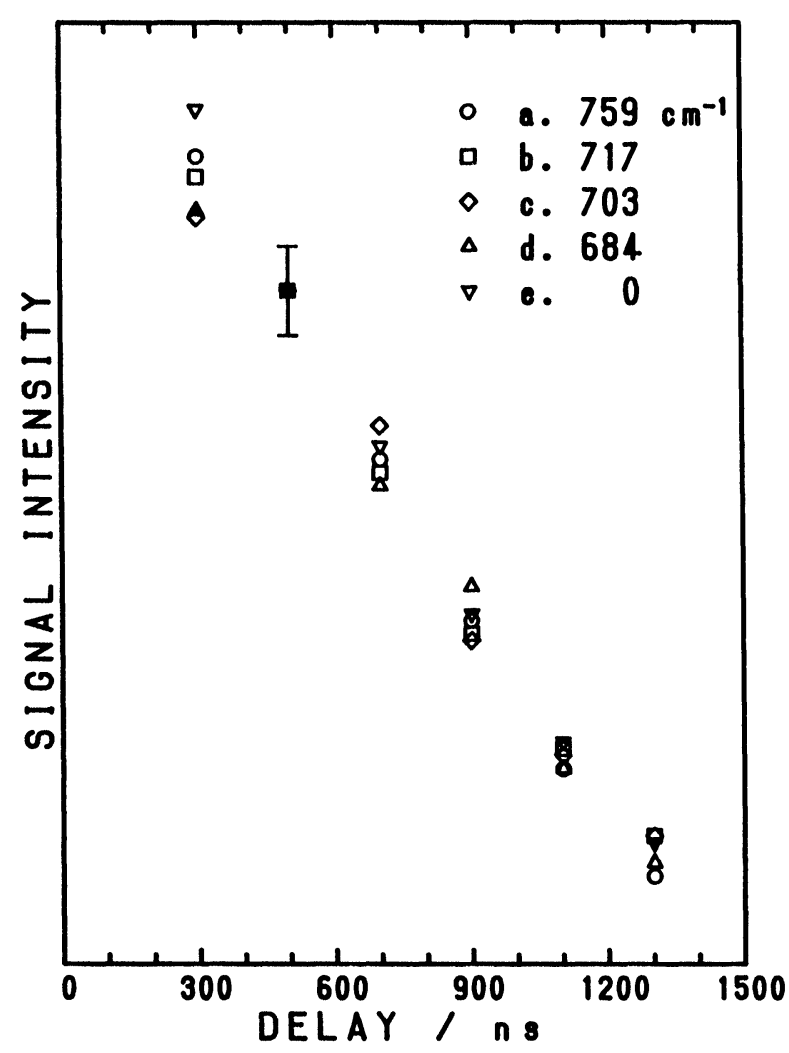

Figure 10 The decay of the intensity of the SEP-induced bands observed with the $786 \mathrm{~cm}^{-1}$ excitation. Marks a-d correspond to the SEP-induced bands a-d shown in Figure 9(b). The mark e shows the intensity of the SEP-induced band due to the transition to $0^{\circ}$.

possibility that the fast (collisionless) relaxation which terminates within $300 \mathrm{~ns}$ would populate some vibrationally excited states, the transitions from which are observed as the SEP-induced bands without any counterpart in the LIF spectrum.

SEP-LIF spectra measured with the $16 \mathrm{a}_{2}$ and $1_{1}$ excitation are shown in Figure 11 together with the LIF excitation spectrum. The bands marked with asterisk are again the background bands. Although main SEP-induced bands can be correlated with the bands in the LIF excitation spectrum, some weak additional bands in the high frequency region have no counterpart in the LIF excitation spectrum. The latters are again assigned to the molecule in the initially prepared vibrational state for the same reason as in the case of the excitation of the lower frequency vibrations.

It is concluded that the vibrationally excited states of anisole below $997 \mathrm{~cm}^{-1}$ has no fast decay process, although the conclusion is not quite definitive concerning the possibility of fast relaxation. The vibrational state density of anisole is estimated to be $\sim 0.5$ and $\sim 1$ states $/ \mathrm{cm}^{-1}$, respectively, at the vibrational energies of 700 and $1,000 \mathrm{~cm}^{-1}$, which are calculated on the basis of the torsional frequency of $81.5 \mathrm{~cm}^{-1} .{ }^{21}$ Although 


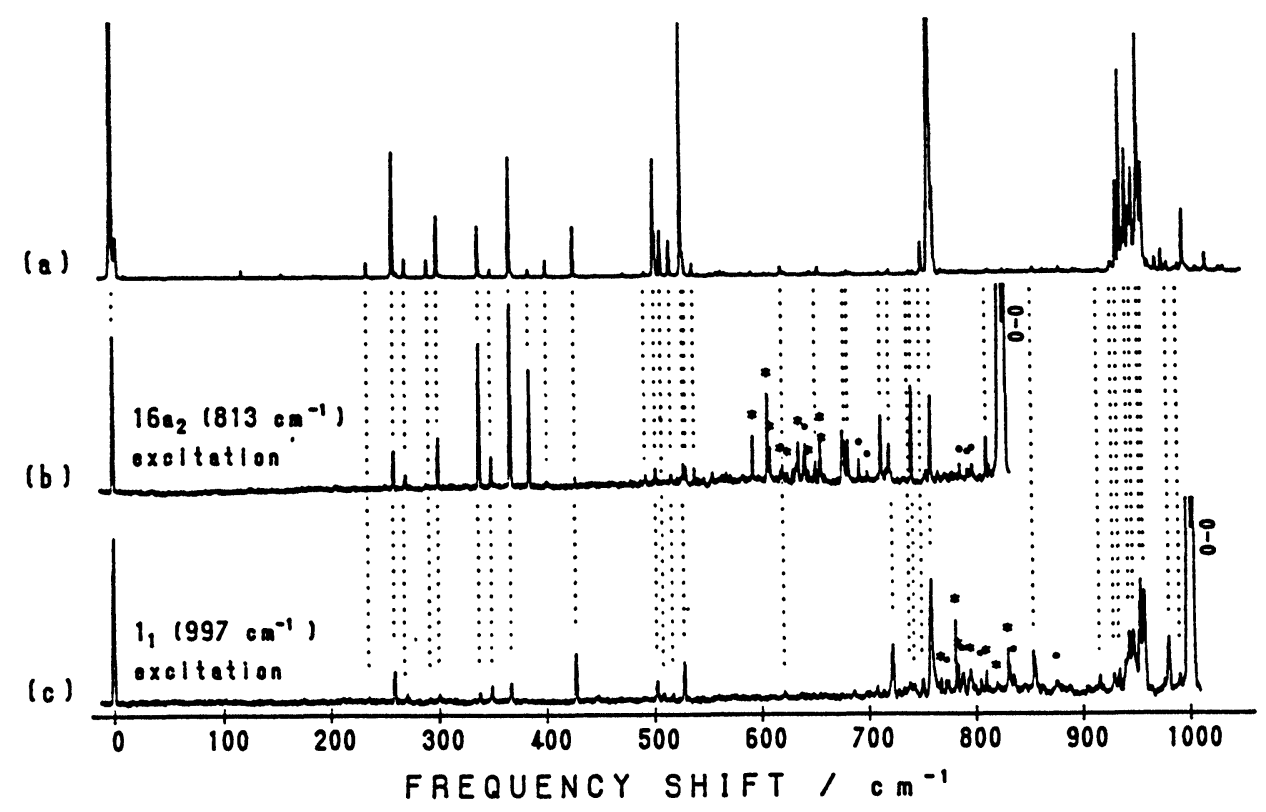

Figure 11 LIF excitation spectrum and SEP-LIF spectra measured with the $16 \mathrm{a}_{2}$ and $1_{1}$ excitation of anisole. Spectra are shifted so that the abscissa shows the excess vibrational energy above $0^{0}$ for each excitation. Bands marked with asterisk are the background bands which are observed without SEP excitation. Those with closed circle appear upon SEP excitation and have no counterpart in the LIF excitation spectrum. The intense bands marked with $0-0$ are the $0-0$ bands.

these values are higher than that of phenol, it might be still insufficient to cause the effective coupling of vibrational states for fast relaxations such as IVR.

\section{CONCLUSION}

SEP-LIF spectra of phenol and anisole were measured to investigate their dynamics in the excited vibrational states. No evidence for IVR in the vibrational states below $1,000 \mathrm{~cm}^{-1}$ in phenol and anisole is found in the time scale employed here. Although the possibility of fast relaxation terminating within $300 \mathrm{~ns}$ could not be ruled out completely, it seems to be most reasonable to conclude that the molecules stay in the initially prepared state after the SEP excitation, in view of the fact that most of the SEP-induced bands have their counterparts in some of the SEP-LIF spectra even if they have no counterpart in the LIF excitation spectrum. This would strongly suggest that they are the transition from the initially prepared state to the common upper state, the transition to which from $0_{0}$ is nearly forbidden.

In spite of our expectation, acceleration of IVR through the torsional motion of the methoxy group of anisole was not observed. It is found that the state density is not sufficiently high for the existence of a single torsional motion with a relatively high frequency. 
As pointed out in Introduction, we have found an evidence for the fast relaxation in the case of the benzonitrile dimer and the benzene-anisole complex. Since their state density is much higher than that of anisole, it seems to be required to investigate a molecule with the intermediate state density to examine whether the state density really determines the relaxation or it is a characteristic feature of van der Waals complexes. In view of this, we are planning to investigate alkylphenylethers with longer alkyl chains, whose state densities are as high as those of the benzonitrile dimer and the anisole•benzene complex.

\section{Acknowledgments}

This research was supported in part by Grant-in-Aid for Encouragement of Young Scientists No. 04740262 from the Ministry of Education, Science and Culture, Japan.

\section{References}

1. R. E. Smalley. J. Phys. Chem., 86, 3504 (1982).

2. J. S. Baskin, M. Dantus and A. H. Zewail. Chem. Phys. Letters, 130, 473 (1986).

3. X. Song, C. W. Wilkerson Jr., J. Lucia, S. Pauls and J. P. Reilly. Chem. Phys. Letters, 174, 377 (1990).

4. C. S. Parmenter. J. Phys. Chem., 86, 1735 (1982).

5. C. S. Parmenter. Faraday Discuss. Chem. Soc., 75, 7 (1983).

6. J. B. Hopkins, P. R. R. Langridge-Smith and R. E. Smalley. J. Chem. Phys., 78, 3410 (1983).

7. T. Suzuki, N. Mikami and M. Ito. J. Phys. Chem., 90, 6431 (1986).

8. T. Ebata and M. Ito. J. Phys. Chem., 96, 3224 (1992).

9. M. Takayanagi and I. Hanazaki. J. Opt. Soc. Am., B 7, 1898 (1990).

10. M. Takayanagi and I. Hanazaki. Chem. Phys. Letters, 190, 115 (1992).

11. M. Takayanagi and I. Hanazaki. J. Chem. Phys., 98, 6958 (1993).

12. M. Takayanagi and I. Hanazaki, unpublished data.

13. A. Sur and P. M. Johnson. J. Chem. Phys., 84, 1206 (1986).

14. R. J. Lipert, G. Bermudez and S. D. Colson. J. Phys. Chem., 92, 3801 (1988).

15. H. D. Bist, J. C. D. Brand and D. R. Williams. J. Mol. Spectrosc., 21, 76 (1966).

16. H. D. Bist, J. C. D. Brand and D. R. Williams. J. Mol. Spectrosc., 24, 413 (1967).

17. W. J. Balfour. Spectrochim. Acta., 39A, 795 (1983).

18. W. J. Balfour. J. Mol. Spectrosc., 109, 60 (1985).

19. F. Lahmani, C. Lardeux-Dedonder, D. Solgadi and A. Zehnacker. J. Phys. Chem., 93, 3984 (1989).

20. T. Ebata, M. Furukawa, T. Suzuki and M. Ito. J. Opt. Soc. Am., B 7, 1890 (1990).

21. H. Konschin, H. Tylli and C. Grundfelt-Forsius. J. Mol. Struct., 77, 51 (1981). 